\title{
L'enseignement technique et professionnel en
} France 1800-1919

Essai de bilan historiographique

Philippe Marchand

\section{(2) OpenEdition}

\section{Journals}

Édition électronique

URL : https://journals.openedition.org/tc/1343

DOI : $10.4000 /$ tc. 1343

ISSN : 1952-420X

\section{Éditeur}

Éditions de l'EHESS

Édition imprimée

Date de publication : 1 juin 2005

ISSN : 0248-6016

\section{Référence électronique}

Philippe Marchand, «L'enseignement technique et professionnel en France 1800-1919», Techniques \& Culture [En ligne], 45 | 2005, mis en ligne le 22 mai 2008, consulté le 29 septembre 2022. URL : http:// journals.openedition.org/tc/1343; DOI : https://doi.org/10.4000/tc.1343

Ce document a été généré automatiquement le 29 septembre 2022.

Tous droits réservés 


\title{
L'enseignement technique et professionnel en France 1800-1919
}

\author{
Essai de bilan historiographique
}

Philippe Marchand

1 L'histoire de l'enseignement technique et professionnel est longtemps restée «un chantier déserté » en histoire de l'éducation ${ }^{1}$. L'état des lieux dressé en 1989 par Pierre Caspard montrait que de 1950 à 1989, les publications traitant de l'histoire de l'enseignement technique ne représentaient que 2 à $3 \%$ des publications en histoire de l'éducation. Son absence, ou sa part très réduite dans les histoires générales de l'enseignement ${ }^{2}$ et dans les histoires générales du travail ou du monde du travail ${ }^{3}$ publiées dans ces mêmes années, est d'ailleurs significative de cet état de choses.

2 Dans les années 1980, le chercheur s'intéressant à l'histoire de l'enseignement technique et professionnel au xix ${ }^{e}$ siècle ne pouvait consulter que les synthèses, anciennes, de P. Astier et L. Cuminal (1909), de J.-H. Cagninacci (1910), de J.-P. Guinot (1946) qui, en dépit de leur ancienneté, surtout pour les deux premières, constituent toujours des ouvrages de référence. Est venu s'ajouter en 1985 l'ouvrage de Bernard Charlot et Madeleine Figeat (1985), bien documenté, mais dont l'approche est beaucoup trop idéologique. L'enseignement technique et professionnel est systématiquement présenté comme l'instrument de reproduction des inégalités sociales. Les choses ont commencé à bouger dans les années 1980-1990. Une équipe de recherche, dirigée par Thérèse Charmasson, se constitue au sein du Service d'histoire de l'éducation, avec pour ambition l'étude des enseignements industriels, commerciaux et agricoles ${ }^{4}$. La publication en 1989 d'un numéro spécial de la revue Formation-Emploi renouvelle en profondeur notre vision de l'histoire de l'enseignement technique et professionnel (Tanguy 1989). Depuis 1990, séminaires, journées d'études ${ }^{5}$, colloques (Bodé \& Marchand 2003, Charmasson 2005) et publications (Pelpel \& Troger 1993) se multiplient. Le service d'histoire de l'éducation organise depuis 2003 un séminaire "Histoire de l'enseignement technique ${ }^{6}$. Ces différentes manifestations de l'intérêt pour ce champ de recherches justifient le constat de Pierre Caspard dans son avant- 
propos aux actes du colloque Formation professionnelle et apprentissage $\mathrm{XVIII}^{e}-\mathrm{XX} \mathrm{X}^{e}$ siècles qui s'est tenu en 2001 :

«Ayant écrit, il y a une quinzaine d'années, que l'histoire de l'enseignement technique était un "chantier déserté", et ayant alors tenté d'en donner les raisons, il m'est agréable d'esquisser aujourd'hui la démonstration en quelque sorte inverse. »

3 Dans ces quelques pages, on présentera d'abord les chantiers largement défrichés au cours des deux dernières décennies, puis on évoquera quelques zones d'ombre réclamant de nouvelles recherches, dont certaines sont en cours.

Les chantiers défrichés

4 En 1987, Thérèse Charmasson, Anne-Marie Lelorrain et Yanick Ripa publient un ouvrage (1987) qui recense 262 textes officiels, les sources répertoriées par lieux de conservation (archives nationales, archives départementales pour huit départements, archives municipales pour trois grandes villes, archives de deux chambres de commerce) et la bibliographie (textes réglementaires et annuaires, sources imprimées et textes historiques). Ainsi conçu, c'est un précieux outil de travail pour tous ceux qui s'intéressent à l'histoire de l'enseignement technique et professionnel. Bien entendu, les textes officiels ne constituent pas la source unique de l'histoire de cette catégorie d'enseignement. Ils ne doivent pas faire illusion, car ils donnent une description incomplète des réalités scolaires. Il n'en reste pas moins que leur consultation est indispensable pour appréhender les actes du pouvoir central dans l'édification d'un système d'enseignement. Le chercheur doit en tenir compte pour situer les informations qu'il rencontre eu égard à la norme définie par l'État. Ajoutons qu'une éclairante mise en perspective historique introduit l'ouvrage, faisant la synthèse des grandes phases du développement de ce type de formation, de la Révolution à l'instauration de la taxe d'apprentissage (1926) consécutive à la loi Astier (1919). Ce survol souligne la spécificité de cet enseignement qui, contrairement aux autres ordres d'enseignement-primaire et secondaire-, n'est pas né d'un principe fondateur exprimé dans un cadre législatif unitaire et cohérent. L'enseignement technique et professionnel n'a eu ni son Napoléon, ni son Guizot, ni son Jules Ferry; il naît de l'amalgame progressif d'établissements hétérogènes qui s'unifient tardivement. On relèvera aussi dans cette introduction les deux grandes tendances qui caractérisent son développement au XIX siècle. D'une part - et la bibliographie le montre bien-, il faut noter les nombreuses initiatives locales et l'absence d'impulsions de l'État, au moins au niveau de l'enseignement technique moyen et inférieur. D'autre part, on constate une emprise croissante de l'État qui cherche à fixer un cadre réglementaire avec un partage des rôles, souvent conflictuel, au sein des instances étatiques (ministère de l'Instruction publique, ministère du Commerce et de l'Industrie). On a donc là deux modèles qui n'ont guère fait l'objet d'une synthèse, au moins jusqu'en 1919 (loi Astier). Enfin, dernière remarque : l'ouvrage porte essentiellement sur l'histoire institutionnelle et administrative de l'enseignement technique. Aussi laisse-t-il de côté tout ce qui relève de l'initiative privée, dont le foisonnement est encore fort mal connu.

5 L'enseignement technique et professionnel de niveau supérieur constitue indiscutablement le noyau dur de l'historiographie actuelle. De nombreux travaux ont contribué à l'approfondissement de nos connaissances dans ce domaine : Terry Shinn (1980), Bruno Belhoste (2003) pour l'École polytechnique, Antoine Picon (1992) pour l'École des ponts et chaussées, Charles Rodney Day (1991) pour les écoles des arts et métiers; également, les publications d'André Grelon $(1989,2003)$ sur le rôle des 
universités dans le développement de l'enseignement technique supérieur, les colloques dirigés par André Thépot (1985), par André Grelon et Françoise Birck (1998) sur les ingénieurs. Genèse et mutations, organisation, procédures de recrutement et de certification, voire population scolaire de ces institutions supérieures sont maintenant bien connues. Cela ne signifie pas, comme on le verra plus loin, qu'il n'y a plus rien à apprendre sur le niveau supérieur.

6 L'histoire des politiques de formation technique et professionnelle est un autre secteur bien défriché. Qui est à leur origine? Qui les impulse? Qui les finance? Qui les contrôle? Quelles sont les relations de pouvoir s'instaurant entre les différents acteurs? Ce questionnement irrigue de nombreux travaux qui ont fait litière de l'image d'un État, principal instigateur du développement de l'enseignement technique et professionnel au XIX ${ }^{\mathrm{e}}$ siècle. De nombreuses publications (Grelon pour l'enseignement supérieur, 1989, 2003 ; Vandenbussche 1984 ; Chapoulie 1989; Briand 1989 ; Suteau 1999 a et b ; Rappe 1997 ; Lacombrade 2003 ; Savoie 1995 ; Pigenet 1990 ; Vanacker-Dhorme 1985, Marchand 2005 ; Bodé 2005, pour les niveaux moyen et inférieur) attestent de la diversité des initiatives. Sociétés scientifiques, sociétés confessionnelles, chambres de commerce, industriels, directeurs d'établissements d'enseignement technique, administrations locales, personnalités, interviennent à des titres divers dans la création et le développement des dispositifs de formation technique et professionnelle. On doit cette mise en évidence à la multiplication des études locales. Il convient d'ailleurs de souligner la fécondité de la démarche monographique qui, seule, rend compte de la complexité de la réalité et permet d'éviter les généralisations hâtives. C'est pour cette raison qu'il faut souhaiter la publication, sous une forme abrégée, des mémoires de maîtrise consacrés aux approches locales. Mais comme le soulignent Gérard Bodé et Philippe Savoie (1995) :

«Le choix de l'échelon local ne dispense pas leurs auteurs de placer leur travail dans une perspective plus large, par la comparaison avec d'autres exemples du même ordre, et par la prise en compte du mouvement général commandé par l'action de l'État, la dynamique des réseaux scolaires, les grands débats nationaux et l'évolution sociale."

Les zones d'ombre

7 Alors que l'offre d'enseignement est bien connue pour les niveaux primaire et secondaire, aucune pesée globale fondée sur le recensement des établissements, des élèves et des enseignants n'a été réalisée pour l'enseignement technique et professionnel. On ne dispose actuellement que des données rassemblées par Louis Christin pour les années 1893-1914 (1968). Et encore ne concernent-elles que les écoles pratiques de commerce et d'industrie, les écoles nationales professionnelles et les écoles de commerce, laissant de côté nombre de lieux de formation qui relèvent de l'initiative tant publique que privée. Que deviennent, par exemple, les élèves fréquentant les enseignements professionnels donnés dans les écoles primaires supérieures?

8 Pour combler cette lacune, le Service d'histoire de l'éducation a lancé en 1989 une grande enquête qui prend l'établissement scolaire comme unité de recherche et le cadre départemental pour le rassemblement des données ${ }^{7}$. Elle doit aboutir à la réalisation de plusieurs atlas-répertoires départementaux. Lieu de convergence de moyens humains, financiers et matériels, lieu de régulation de flux d'élèves, lieu enfin de caractéristiques conditionnant son encadrement et son organisation pédagogique, l'établissement scolaire permet de relativiser le poids et l'impact des impulsions 
officielles. Dans cette enquête, est considérée comme établissement de formation technique et professionnelle toute cellule qui, quels que soient son statut administratif (public, privé), sa structure (établissement autonome, annexé) ou sa taille, délivre des connaissances techniques et professionnelles utilisables dans les domaines industriel, commercial, agricole et vétérinaire. Sont ainsi retenues des écoles qui à première vue semblent échapper à cette définition, mais qui, après inventaire des formations dispensées, méritent de figurer dans les répertoires : écoles pour aveugles, pour sourds et muets, écoles ménagères, écoles militaires ne se contentant pas de former des militaires mais donnant des formations monnayables sur le marché du travail (Le Gallo 2005). Plus d'une vingtaine de départements constituent actuellement le terrain d'enquête. À la lumière des résultats déjà obtenus, il apparaît que l'offre d'enseignement technique et professionnel est beaucoup plus importante et diversifiée qu'on ne le soupçonnait, en particulier aux niveaux moyen et inférieur. Un premier atlas-répertoire consacré au département de la Corrèze est paru (Bodé \& Veynes 2004).

Si l'histoire des diplômes de l'enseignement technique et professionnel est maintenant bien connue pour les niveaux moyen et inférieur grâce à l'ouvrage de Guy Brucy (1998), grâce aussi aux ouvrages cités plus haut pour le niveau supérieur, en revanche, on ignore encore tout, ou presque tout, des contenus d'enseignement, de la pédagogie mise en œuvre, des manuels utilisés dans l'enseignement technique et professionnel. On n'a pas encore étudié systématiquement la part accordée à l'enseignement de culture générale, à l'enseignement théorique, à l'enseignement pratique. Rien n'a été fait, par exemple, pour étudier l'application du chapitre $\mathrm{V}$ Enseignement et bourses du décret du 22 février 1892 qui organise les écoles pratiques de commerce et d'industrie en laissant à leurs conseils de perfectionnement, où figurent des représentants du monde économique, le soin d'élaborer le programme des enseignements et l'emploi du temps. On doit admettre que l'historien se heurte ici à une double difficulté. Tout d'abord, il existe un véritable déficit de sources, sans doute plus important dans ce domaine que pour l'enseignement secondaire ou pour l'enseignement primaire. La seconde difficulté est celle de la technicité de ce type d'enseignement. Les compétences et les connaissances qu'il transmet ne font guère partie du bagage intellectuel des historiens. Il faut donc que ceux qui s'engagent dans cette voie collaborent avec les personnes qui ont reçu la formation technique spécifique.

10 Parmi les lacunes historiographiques, déjà relevées par Lucie Tanguy en 1989, il faut faire une place particulière au cas des filles, dont la méconnaissance a été longtemps une conséquence de la sous-estimation de la place des femmes dans le monde et la division du travail. Ce retard tend à se combler, grâce en particulier aux travaux réalisés sous la direction de Sylvie Schweitzer au sein du Centre Pierre Léon de l'Université Lyon II. Les résultats en sont publiés sous une forme condensée dans les bulletins du Centre ${ }^{8}$. C'est, sans doute, le domaine de l'enseignement technique et professionnel au féminin qui nécessite une identification précise des lieux de formation en ne se limitant pas seulement aux écoles dites "professionnelles» et aux écoles pratiques de commerce et d'industrie. Comme le montrent Jean-Pierre Briand et JeanMichel Chapoulie (1992 ${ }^{9}$ ), Jacqueline Claire et Sylvie Schweitzer (2003), S. Court (1999), il faut regarder du côté des écoles primaires supérieures et des cours complémentaires qui dispensent à des milliers de filles un enseignement pratique assurant des débouchés vers le commerce et l'industrie. Il faut aussi tenir compte des écoles privées qui forment aux métiers du secrétariat et qui se multiplient dans les grandes villes à la fin du siècle. Enfin, n'oublions pas les orphelinats, les ouvroirs, les écoles ménagères, les 
cours de couture. Mais quid des formations dispensées dans ce type d'établissements? Préparent-ils leurs élèves à devenir de bonnes épouses, de bonnes mères de famille et/ ou à l'exercice d'un métier ? Les recherches de Daniel Murat (2002) montrent que, très tôt, la notion de formation professionnelle tient une place centrale dans les stratégies pédagogiques mises en œuvre au sein des institutions fermées pour filles des départements de l'Hérault, du Gard et du Vaucluse.

11 Dans un ouvrage publié en 1990, Claude Dubar écrit : «À la fin du siècle (XIX)e, la grande majorité des ouvriers et des paysans n'a droit à aucune formation professionnelle ni initiale ni continue» (1999: 12, souligné par nous). Or, les études d'Astier et Cuminal (1909) et de Cagninacci (1910) -qui ne figurent pas dans la bibliographie de l'ouvragemettaient en lumière l'existence, dans les dernières décennies du XIX siècle, de nombreux cours de formation technique et professionnelle destinés aux adultes. Précisons que l'« adulte » ne se définit pas, ici, par l'âge (Terrot 1983). L'adulte est celui qui, ayant échappé aux mailles du filet scolaire ou ayant abandonné l'école à un quelconque stade, s'inscrit dans une activité de formation technique et professionnelle. Ces formations ne doivent pas être confondues avec les « cours d'adultes » qui peuvent offrir des éléments d'enseignement pratique, mais dans un contexte qui relève des objectifs de l'enseignement primaire. Enfin, les cours professionnels destinés aux adultes concernent principalement les ouvriers et les employés, c'est-à-dire les gros bataillons de l'armée industrielle. Le vide historiographique, que ne comblent pas quelques études ponctuelles, s'avère préjudiciable à une connaissance approfondie des processus de formation des ouvriers et des employés ainsi que des possibilités de qualification (Marchand 2001).

12 Les informations collectées dans l'enquête sur les établissements scolaires devraient permettre d'affiner notre vision des rapports du patronat et des organisations ouvrières avec la formation technique et professionnelle. En ce qui concerne l'attitude du patronat, des études récentes (Bodé 2003; Kharaba 2003; Lacombrade 2003; Marchand 2003) soulignent son évolution au cours du siècle. Indiscutablement, le dénigrement de la scolarisation de la formation des ouvriers perdure tout au long $d u$ $\mathrm{XIX}^{\mathrm{e}}$ siècle. Les arguments sont récurrents : l'enseignement est jugé trop théorique, développant chez les futurs ouvriers et employés une mauvaise perception de leurs aptitudes et de leurs capacités, et formant une main-d'œuvre inadaptée aux postes de travail qu'elle aura à occuper, et surtout indocile. À l'inverse, et cette attitude est contemporaine du développement de la grande industrie, le patronat commence à voir dans la formation au sein du système scolaire un moyen de lutter contre les " mauvaises habitudes que prennent les ouvriers dans les usines» (Marchand 2003). Il serait cependant réducteur d'y voir seulement une volonté de contrôle de la maind'œuvre. Le patronat considère la formation initiale et continue comme le moyen de s'attacher la main-d'œuvre qualifiée dont il a besoin, comme le moyen aussi de consolider le lien existant entre la communauté ouvrière et l'entreprise. Mais le patronat ne l'accepte qu'à la condition d'en exercer le contrôle, partiel ou total, ce qui l'incite à fonder ses propres lieux de formation et à participer à l'administration des écoles pratiques de commerce et d'industrie.

Qu'en est-il du rapport des organisations ouvrières à l'enseignement technique et professionnel ? Quel rôle ont-elles joué dans son développement? Encore une question fort mal connue, et qui n'a guère suscité de communications lors du colloque Formation professionnelle et ouvrière, $\mathrm{XVIII}^{e}$-XXe siècles, conséquence sans doute de la désaffection 
actuelle pour l'histoire du monde ouvrier. L'ouvrage de Cagninacci (1910: 281) ouvre une piste, au moins en ce qui concerne les cours professionnels pour adultes, en soulignant le désintérêt croissant des organisations ouvrières pour ce type de formation. Cette idée est reprise par J.-P. Guinot (1946). Selon lui, les organisations ouvrières auraient créé de nombreux cours professionnels avant 1884 pour se voir accorder le droit d'exister. Elles auraient poursuivi leur action quelques années encore après leur reconnaissance officielle, puis auraient laissé ce soin aux bourses du travail. Une recherche en cours sur l'attitude des syndicats ouvriers du département du Nord confirme la problématique de Cagninacci pour la période à laquelle il se réfère. De 1890 à 1914, les syndicats ont dû répondre à un questionnaire préfectoral qui comportait une question sur leur participation à l'organisation de cours professionnels. En 1906, le préfet du Nord fait dresser un état des cours professionnels organisés à la demande des syndicats ouvriers. L'analyse de cette documentation révèle que parmi la centaine de syndicats ouvriers recensés dans le département, deux seulement-le syndicat des imprimeurs de Lille et le syndicat des mécaniciens de Tourcoing-, se sont impliqués dans la création de cours professionnels pour adultes. On notera d'ailleurs que les discours sur la formation technique et professionnelle tenus dans ces années 1880-1914 ne sont produits que par des responsables politiques (à l'exception des socialistes), économiques et administratifs. On remarquera aussi que les syndicats ouvriers invités à déposer lors de l'Enquête parlementaire sur l'état de l'industrie textile et la condition des ouvriers tisseurs de 1903-1904 n'abordent pas cette question (Marchand 2003). De son côté, Daniel Rappe (1997) souligne la place occupée par la Bourse du travail de Lyon dans le développement des cours professionnels. Si la Bourse du travail de Lyon se sent concernée par cette question, les syndicats, en revanche, ont une attitude nettement plus frileuse. Une enquête diligentée en 1895-1896 signale que huit syndicats seulement sur soixante-deux souhaitent l'organisation de cours de perfectionnement relatifs à leur profession. Plusieurs questions méritent donc d'être approfondies. Peut-on établir dans le rapport des organisations ouvrières à la formation professionnelle et technique cette césure chronologique correspondant à la reconnaissance officielle des syndicats ? L'enquête doit être reprise en amont de la loi de 1884 sur les syndicats professionnels. Faut-il, comme Guinot le soutient, retenir le seul argument financier pour expliquer la position en retrait des syndicats à partir de 1884 ? Ne conviendrait-il pas de tenir compte de leur méfiance à l'égard de formations accusées de faire le jeu du patronat et susceptibles de produire des individus qualifiés qui ne se percevront plus ou ne seront plus perçus comme appartenant à la classe ouvrière ? Cette hypothèse mériterait d'être vérifiée.

D'autres zones d'ombre subsistent. On ne sait toujours pas grand-chose de l'articulation entre la formation et l'économie. Doit-on considérer que la création de formations techniques et professionnelles anticipe ou accompagne les demandes du marché et les besoins de la consommation? Quelques études abordent cette question pour les niveaux supérieurs. On a longtemps cru, par exemple, que la création des instituts techniques des facultés des sciences, à la fin du siècle, devait beaucoup aux demandes industrielles locales. Les auteurs de la recherche "Ville et institutions scientifiques » révèlent que :

«Les industriels sont loin d'être les initiateurs de ce mouvement. Ce sont au contraire les universitaires qui y tiennent le rôle principal, les entrepreneurs ne faisant que suivre et soutenir (éventuellement financièrement) des projets à la formation desquels ils contribuent peu. » (Grelon 1989) 
15 toujours dans l'urgence pour créer les formations susceptibles de doter le système
productif d'agents compétents et innovants dans le domaine de la mode (Marchand \& Terrier 2005). Qu'en est-il des formations de niveaux moyen et inférieur?

Le développement de la formation technique et professionnelle scolarisée ne doit pas faire oublier la question de l'apprentissage au sens traditionnel du terme, c'est-à-dire la transmission d'un métier ou des gestes d'un métier par le maître à son apprenti. Un discours récurrent sur « la crise de l'apprentissage » provoquée par le développement de l'industrie et de procédés techniques de plus en plus complexe traverse tout le XIX siècle. L'apprentissage aurait été confiné dans le milieu de l'artisanat. Yves Lequin (1986) montre que le développement de la grande industrie et des modes d'organisation du travail ne fait pas disparaître la circulation des savoirs à l'intérieur de l'entreprise. Les situations d'apprentissage, au sens le plus classique du terme, survivent en s'adaptant aux changements matériels et aux nouveaux impératifs de la production. L'enquête sur l'apprentissage doit être reprise pour affiner notre vision de la constitution des identités ouvrières ainsi que des résistances du monde ouvrier à la formation scolarisée.

Les personnalités marquantes du monde de l'éducation, du monde économique et de la sphère politique investies dans la formation technique et professionnelle sont encore peu étudiées. On dispose de quelques comparaisons historiques entre les différents systèmes d'enseignement technique et professionnel à l'œuvre en Allemagne, en Belgique et dans les pays anglo-saxons (Bodé 2003 ; Chézeau 2003 ; Grootaers 2003). Souhaitons toutefois que ces comparaisons soient étendues à des pays comme l'Italie où les recherches sur l'histoire de l'enseignement technique et professionnel au XIX ${ }^{\mathrm{e}}$ siècle témoignent d'une grande vitalité ${ }^{10}$.

$\mathrm{Au}$ terme de cette présentation des acquis et des chantiers de recherches futurs, il convient de s'arrêter sur deux constatations : l'histoire de l'enseignement technique et professionnel est une histoire en construction que d'autres colloques et publications viendront enrichir. À cet égard, l'enquête menée par le Service d'histoire de l'éducation devrait fournir l'ensemble des données qui permettront d'appréhender un système d'enseignement dans toute sa richesse et sa complexité. En tenant compte de la dimension pédagogique des transformations du travail et de la production, l'histoire de l'enseignement technique et professionnel témoigne de sa validité comme composante de l'histoire économique et sociale.

\section{BIBLIOGRAPHIE}

Albertini, Pierre

1992. L'École en France, $\mathrm{XIX}$ - $-\mathrm{XX}$ e siècles, de la maternelle à l'Université. Paris : Hachette. 
Astier, P. \& et L. Cuminal

1909. L'enseignement technique industriel et commercial en France et à l'étranger. Paris : Dunod et Pinat éditeurs (2ème édition augmentée, Paris, 1912).

Belhoste, Bruno

2003. La Formation d'une technocratie L'École polytechnique et ses élèves de la Révolution au Second Empire. Paris : Belin (« Histoire de l'éducation »).

Bodé, Gérard

2001. «L'enseignement technique en France et en Allemagne : écoles de perfectionnement et cours professionnels, 1820-1940 », pp. 147-160, in Les ouvriers qualifiés de l'industrie.

«Le "modèle" de formation professionnel allemand au XIX ${ }^{\mathrm{e}}$ siècle : naissance d'un malentendu? L'exemple de l'Alsace-Lorraine (1871-1918), pp. 389-418, in Formation professionnelle et apprentissage $\mathrm{XVIII}{ }^{e}-\mathrm{XX}{ }^{e}$ siècles. Actes du colloque international l'Histoire de la formation technique et professionnelle en Europe du XVIII ${ }^{e}$ siècle au milieu du XX $x^{e}$ siècle. Villeneuve d'Ascq : Revue du NordINRP

2005. « Le ministre, le préfet et le proviseur : stratégies d'établissement, politique nationale et contingences locales dans l'enseignement technique, 1800-1940 ", pp. 17-30, in T. Charmasson (ed.), Formation au travail, enseignement technique et apprentissage (CTHS, 127ème congrès, Nancy, 15-20 avril 2002). Paris : Éditions du Comité des travaux historiques et scientifiques.

Bodé, Gérard \& Hubert Veynes

2004. Les Établissements d'enseignement technique en France, 1789-1940. Tome 1 : La Corrèze. Paris : Service d'histoire de l'éducation, INRP.

Bodé, Gérard \& Philippe Marchand (eds)

2003. Formation professionnelle et apprentissage, $\mathrm{XVIII}^{e}-\mathrm{XX}^{e}$ siècles. Actes du colloque international l'Histoire de la formation technique et professionnelle en Europe du XVIII ${ }^{\mathrm{e}}$ siècle au milieu du $\mathrm{xx}^{\mathrm{e}}$ siècle. Villeneuve d'Ascq : Revue du Nord-INRP.

Bodé, Gérard \& Philippe Savoie 1995. «L'approche locale de l'histoire des enseignements techniques et intermédiaires : nécessités et limites ", Histoire de l'éducation $66:$ 5-10.

Briand, Jean-Pierre

1989. "L'apparition du "préapprentissage" dans les grandes villes au début du xx siècle ", pp. 42-64, Formation-Emploi, L'enseignement technique et professionnel Repères dans l'histoire (1830-1960).

Briand, Jean-Pierre \& Jean-Michel Chapoulie

1992. Les Collèges du peuple. L'enseignement primaire supérieur et le développement de la scolarisation prolongé sous la Troisième République. Paris-INRP-ENS-Fontenay Saint-Cloud.

Brucy, Guy

1998. Histoire des diplômes de l'enseignement technique et professionnel (1880-1965). L'État, l'école, les entreprises et la certification des compétences. Paris : Belin.

Cagninacci, J.-H.

1909. L'instruction professionnelle de l'ouvrier. Paris : Librairie nouvelle de droit et de jurisprudence

A. Rousseau.

Caspard, Pierre

1989. « Un chantier déserté. L'histoire de l'enseignement technique », pp. 193-197, Formation

Emploi, L'enseignement technique et professionnel Repères dans l'histoire (1830-1860).

Chapoulie, Jean-Michel

1989. « Deux expériences de création d'établissements techniques au XIX ${ }^{\mathrm{e}}$ siècle », pp. 15-41, 
Formation-Emploi, L'enseignement technique et professionnel Repères dans l'histoire (1830-1960).

Charlot, Bernard \& Madeleine Figeat

1985. Histoire de la formation des ouvriers, 1789-1984. Paris : Minerve.

Charmasson, Thérèse

2005. Formation au travail, enseignement technique et apprentissage (CTHS, Actes du 127ème congrès, Nancy, 15-20 avril 2002). Paris : Éditions du Comité des travaux historiques et scientifiques.

Charmasson, Thérèse, Anne-Marie Lelorrain \& Yanick Ripa

1987. L'enseignement technique de la Révolution à nos jours. Textes officiels avec introduction, notes et annexes. Tome I : De la Révolution à 1926. Paris : INRP-Economica.

Chézeau, Nicole

2003. "Étude comparative de la formation des ingénieurs à la métallurgie dans les pays anglosaxons et en France de 1870 à 1920, pp. 419-428, Formation professionnelle et apprentissage XVIII ${ }^{e}-X^{e}$ siècles. Actes du colloque international l'Histoire de la formation technique et professionnelle en Europe du XVIII ${ }^{e}$ siècle au milieu du XX ${ }^{e}$ siècle. Villeneuve d'Ascq : Revue du Nord-INRP.

Christin, Louis

1974. L'Enseignement technique. Colloque La Scolarisation en France depuis un siècle, pp. 77-114. Paris/ La Haye : Mouton/Publications de l'Université des sciences sociales de Grenoble.

Claire, Jacqueline \& Sylvie Schweitzer

2003. «Des formations initiales pour des métiers : l'enseignement féminin à Lyon, mi-XIX ${ }^{\mathrm{e}}$ sièclemi- XX ${ }^{e}$ siècle ", pp. 175-192, Formation professionnelle et apprentissage XVIII ${ }^{e}$ XX ${ }^{e}$ siècles. Actes du colloque international l'Histoire de la formation technique et professionnelle en Europe du XVIII siècle au milieu du $\mathrm{XX}^{e}$ siècle. Villeneuve d'Ascq : Revue du Nord-INRP.

Court, Sophie

1999. « Deux institutions d'enseignement technique de jeunes filles à Lyon, 1877-1939 », Bulletin du Centre Pierre Léon d'histoire économique et sociale, Formation, emplois, $\mathrm{XIX}^{\mathrm{e}}-\mathrm{XX}^{\mathrm{e}}$ siècles 3-4 :

31.

Dewerpe, Alain

1989. Le Monde du travail en France 1800-1950. Paris : Armand Colin (« Cursus »).

Dubar, Claude

1999. La Formation professionnelle continue. Paris : La Découverte (« Repères », $n^{\circ} 28$ ).

Folhen, Claude \& François Bédarida

1959. Histoire générale du travail, tome III, L’ère des Révolutions (1765-1914). Paris : Nouvelle

Librairie de France.

Grelon, André

1989. « Les universités et la formation des ingénieurs (1870-1914) », pp. 65- 88, Formation-Emploi, L'enseignement technique et professionnel Repères dans l'histoire (1830-1960).

1996. «Villes et institutions scientifiques » Rapport final Juin 1996, COREP. Toulouse, Programme interdisciplinaire de Recherche sur les Villes.

2003. «La naissance des instituts industriels catholiques :le rôle pionnier du Nord de la France (1885-1914 », Cahiers du Centre Pierre Léon d'histoire économique et sociale 2 : 106-114.

Grelon, André \& Françoise Birck (eds)

1998. Des ingénieurs pour la Lorraine. Actes du colloque Enseignements industriels et formations 
technico-scientifiques supérieures Enseignements industriels et formations technicoscientifiques supérieures en Lorraine, $\mathrm{XIX}^{\mathrm{e}}-\mathrm{XX}^{\mathrm{e}}$ siècles. Saint-Julien-lès-Metz : Éditions Serpenoise. Grootaers, Dominique

2003. «Les paradigmes de l'enseignement technique et professionnel masculin durant la période de son premier développement (1860-1940). Comparaison des contextes nationaux belge et français ", pp. 379-488, Formation professionnelle et apprentissage XVIII ${ }^{e}-X X^{e}$ siècles. Actes du colloque international l'Histoire de la formation technique et professionnelle en Europe du XVIII siècle au milieu du $\mathrm{XX}^{e}$ siècle. Villeneuve d'Ascq : Revue du Nord-INRP.

Guinot, Jean-Pierre

1946. Formation professionnelle et travailleurs qualifiés depuis 1789. Paris : Domat-

Montchrestien.

Kharaba, Ivan

2003. « La formation initiale au sein des établissements Schneider au XIX ${ }^{e}$ siècle », pp. 281-288,

Formation professionnelle et apprentissage XVIII ${ }^{e}-\mathrm{XX}^{e}$ siècles. Actes du colloque international l'Histoire de la formation technique et professionnelle en Europe du XVIII ${ }^{e}$ siècle au milieu du XX $x^{e}$ siècle. Villeneuve d'Ascq : Revue du Nord-INRP.

Lacombrade, Philippe

2003. « Le patronat parisien et l'enseignement professionnel à la Belle Époque : modèles européens et modernisation du système français (1902-1914)», Formation professionnelle et apprentissage $\mathrm{XVIII}^{e}-\mathrm{XX}{ }^{e}$ siècles. Actes du colloque international l'Histoire de la formation technique et professionnelle en Europe du XVIII ${ }^{e}$ siècle au milieu du XX $x^{e}$ siècle. Villeneuve d'Ascq : Revue du NordINRP.

Le Gallo, Yolande

2005. « La formation des ouvriers et techniciens des arsenaux de la marine du début du XIX siècle à la fin des années 30 : l'exemple de Toulon ", pp. 177-188, in T. Charmasson (ed.), Formation au travail, enseignement technique et apprentissage (CTHS, Actes du 127ème congrès, Nancy, 15-20 avril 2002). Paris : Éditions du Comité des travaux historiques et scientifiques.

Lequin, Yves

1986. « Apprenticeship in Nineteeth-Century France : A continuing tradition or a break with the past ? ", pp. 457-474, in S. L. Kaplan and C. J. Koepp (eds), Work in France Representations, Meaning, Organization, and Practice. Ithaca/London : Cornwell University Press.

Marchand, Philippe

1995 a. « Une expérience de formation professionnelle d'adultes. Le cours municipal des chauffeurs-mécaniciens de Lille, 1858-1939 ", Histoire de l'éducation 66 : 137-158.

«La qualification en débat parmi les responsables politiques,économiques et éducatifs du Nord 1860-1940 ", pp. 137-146, in G. Gayot et Ph. Minard (eds), Les ouvriers qualifiés de l'industrie (XVI ${ }^{e}-\mathrm{XX}^{e}$ siècle), Formation, emploi, migrations, Revue du Nord, hors série, (« Histoire » ${ }^{\circ} 15$ ).

2003. «Pour une histoire de la formation professionnelle des adultes. Le cas du Nord de la France (milieu XIX ${ }^{e}$ siècle-1914»), pp.157-173, Formation professionnelle et apprentissage XVIII ${ }^{e}-X^{e}$ siècles. Actes du colloque international l'Histoire de la formation technique et professionnelle en Europe du $\mathrm{XVIII}^{e}$ siècle au milieu du XX $\mathrm{X}^{e}$ siècle. Villeneuve d'Ascq : Revue du Nord-INRP

2005. «Les écoles pratiques de commerce et d'industrie dans le Nord de la France, 1892-1940. Jalons pour une histoire nationale de l'enseignement technique et professionnel moyen », pp. 31-52, in T Charmasson (ed.), Formation au travail, enseignement technique et apprentissage (CTHS, Actes du 127ème congrès, Nancy, 15-20 avril 2002). Paris : Éditions du Comité des travaux historiques et scientifiques. 
Marchand, Philippe \& Didier Terrier

2005. « Les exigences de la mode et la formation technique des hommes : les écoles d'art et d'industrie à Roubaix et Tourcoing (fin XIX siècle) », pp. 1195-1203, in G. L. Fontana and G. Gayot (eds), Wool, products and markets (13th-20th century). Padoue : Cleup Scarl.

Mayeur, Françoise

1981. Histoire générale de l'éducation et de l'enseignement en France, tome III De la Révolution à l'école républicaine (1789-1930). Paris : Nouvelle Librairie de France.

Murat, Daniel

2002. «Le travail des enfants abandonnés du Gard dans les filatures de soie (seconde moitié du XIX ${ }^{\mathrm{e}}$ siècle) », pp. 153-168, in R. Caty (ed.), Enfants au travail, attitudes des élites en Europe occidentale et méditerranéenne, aux $\mathrm{XIX}^{e}$ et $\mathrm{XX}^{e}$ siècles. Aix-en-Provence : Publications de l'Université de Provence (« Le temps de l'histoire »).

Noiriel, Gérard

1986. Les Ouvriers dans la société française $\mathrm{XIX}^{\mathrm{e}}-\mathrm{XX}^{\mathrm{e}}$ siècle. Paris : Éditions du Seuil.

Pelpel, Patrice \& Vincent Troger

1993. Histoire de l'enseignement technique. Paris : Hachette.

Ponteil, Félix

1966. Histoire de l'enseignement en France Les grandes étapes, 1789-1964. Paris : Sirey.

Picon, Antoine

1992. La Naissance de l'ingénieur moderne. L'école des ponts et chaussées, 1747-1851. Paris :

Presses de l'ENPC.

Pigenet, Michel

1990. «L'ENP de Vierzon et le problème de la formation professionnelle dans une ville ouvrière (années 1880-1914)», Revue historique, tome CCLXXXII/2, 572 : 367-389.

Prost, Antoine

1968. Histoire de l'enseignement en France, 1860-1967. Paris : Armand Colin.

Shinn, Terry

1980. Savoir scientifique et pouvoir social : l'École polytechnique, 1794-1914. Paris : Presses de la fondation nationale des sciences politiques.

Tanguy, Lucie (coordonné par)

1989. «L'enseignement technique et professionnel. Repères dans l'histoire (1830-1960) », Formation Emploi 27-28.

Terrot

1983. Histoire de l'éducation des adultes. Paris : Edilig.

Thépot, André (ed.)

1985. L'Ingénieur dans la société française. Colloque l'Écomusée du Creusot, 23 au 25 octobre 1980. Paris : Éditions ouvrières.

Rappe, Daniel

1997. «Les cours professionnels de la Bourse du travail de Lyon (1896-1914) », Bulletin du Centre Pierre Léon d'histoire économique et sociale 3-4 :39-49.

Savoie, Philippe

1995. «Offre locale et engagement de l'État. Les enseignements technique et primaire supérieur à 
Nancy et les conditions de leur évolution sous la Troisième République ", Histoire de l'éducation 66, L'offre locale d'enseignement $\mathrm{XIX}^{e}$-XXe siècles : 47-84.

Suteau, Marc

1999a. Une ville et ses écoles, Nantes, 1830-1940. Rennes : Presses Universitaires de Rennes.

1999b. « Le rôle des villes, du patronat et des chefs d'établissements dans le développement des écoles techniques : l'exemple des écoles municipales de Nantes de 1890 à 1940 ", Le Mouvement Social $189: 67-82$.

Vandenbussche, Robert

1984. «Les pouvoirs locaux et les débuts de la formation professionnelle dans le Nord sous la Troisième République (1880-1914)», Revue du Nord LXVI/261-262 : 883-892.

Vanacker-Dhorme, Catherine 1985. «Les débuts de l'enseignement professionnel dans le Nord : l'exemple de l'ENP d'Armentières ", Revue du Nord, Maîtres et écoles dans le nord de la France contemporaine LXVII-266 : 749-768.

\section{ANNEXES}

Repères chronologiques

$1794-1919$

1ère période

-6 frimaire an 3 (26 novembre 1794) : décret portant création de l'Ecole centrale des Travaux publics (Ecole polytechnique à partir du 15 fructidor an III ( $1^{\text {er }}$ septembre 1795).

-19 vendémiaire an III (10 octobre 1794) : décret établissant un Conservatoire des arts et métiers à Paris.

-30 vendémiaire an IV (22 octobre 1795) : décret organisant les « écoles spéciales » en écoles d'application de l'Ecole polytechnique.

2ème période

-2 août 1816 : création de l'École des mines de Saint-Étienne.

-création de l'École spéciale de commerce e Paris.

-31 août 1828 : ordonnance exprimant la volonté de rattacher et de coordonner les diverses institutions publiques destinées à l'enseignement des arts industriels. -1829 : création de l'École centrale des Arts et Manufactures.

-26 mars 1829 : ordonnance autorisant les chefs de pension ou d'institution à donner dans leurs établissements l'instruction qui convient plus particulièrement aux professions industrielles et manufacturières -1830 : école établie par la Société industrielle de Nantes.

-28 juin 1833 : création de l'enseignement primaire supérieur avec dessin linéaire, arpentage.

-29 octobre 1833 : création de l'école de la Martinière à Lyon.

-9 octobre 1838 : enquête générale du ministère des Travaux publics, de l'Agriculture et du Commerce visant au recensement des établissements formés pour les arts industriels.

-30 juin 1843 : création d'une école des Arts et Métiers à Aix-en-Provence. 
-22 septembre 1843 : ordonnance royale instituant une école pratique destinée à la formation des maîtres-ouvriers mineurs.

-5 mars 1847 : statut sur l'enseignement des collèges introduisant à partir de la troisième un enseignement des éléments de comptabilité général, de droit commercial et d'économie agricole.

-29 mars 1848 : installation d'une Commission de professeurs du Conservatoire des Arts et Métiers chargée de l'étude d'un système général pour l'enseignement des sciences appliquées aux arts industriels.

-22 février 1851 ; loi sur l'apprentissage.

-1854 : fondation de l'école professionnelle de Mulhouse.

-14 juin 1862 : installation d'une commission chargée d'étudier la création d'un enseignement professionnel dans les lycées.

-22 juin 1863 : installation par le ministère de l'Agriculture, du Commerce et des Travaux publics d'une commission chargée de rechercher les moyens de développer un enseignement professionnel.-2 octobre 1863 : instruction du ministre de l'Instruction publique relative à l'établissement d'un enseignement secondaire dit professionnel dans les lycées.

-30 novembre 1863 : décret portant création de l'École nationale d'horlogerie de Cluses. -20 juin 1865 : présentation par la Commission de l'enseignement professionnel d'un rapport préconisant l'adoption pour l'enseignement technique d'une organisation générale tout en retenant l'idée que toute liberté soit laissée aux fondateurs d'établissements.

-15 février 1867 : projet de loi relatif à l'enseignement technique repoussé. -15 juillet 1867 : création d'une école professionnelle municipale au Havre -1873 : création d'une école municipale des apprentis de Paris -31 mars 1878 : décret portant création de l'École des maîtres-ouvriers mineurs de Douai.

3ème période

-11 décembre 1880 : loi relative aux écoles manuelles d'apprentissage.

-9 juillet 1881 : décret portant création à Vierzon d'une école nationale d'enseignement primaire supérieur et d'enseignement professionnel préparatoire à l'apprentissage. -30 juillet 1881 : décret relatif à l'organisation des écoles manuelles d'apprentissage. Institution de la double tutelle ministère de l'Instruction publique et du ministère du Commerce et de l'industrie

-9 juillet 1883 : circulaire du ministère du Commerce et de l'Industrie prescrivant une enquête sur l'enseignement technique à ses divers degrés.

-28 septembre 1885 : circulaire du ministre de l'Instruction publique prescrivant une enquête sur l'enseignement technique à ses divers degrés.

-15 mars 1886 : première réunion de la commission mixte (Instruction publique, Commerce et Industrie) chargée d'étudier les problèmes posés par la loi du 11 décembre 1880.

-30 octobre 1886 : loi sur l'organisation de l'enseignement primaire plaçant les écoles d'apprentissage au nombre des écoles primaires.

-22 novembre 1886 : circulaire du ministère de l'Instruction publique et du ministère du Commerce et de l'Industrie lançant une enquête pour recenser tous les établissements publics ou privés dans lesquels une part est faite à l'enseignement technique ou professionnel. 
-6 juillet 1887 : Projet de loi sur l'enseignement technique

-17 mars 1888 : décret établissant le régime du condominium (double autorité du ministère de l'Instruction publique et du ministère du Commerce et de l'Industrie) sur les écoles manuelles d'apprentissage et sur les écoles d'enseignement primaire supérieur ou complémentaire comprenant des classes ou des cours d'enseignement professionnel.

-26 janvier 1892 : loi de finances provoquant le rattachement des écoles primaires supérieures professionnelles dont l'enseignement est principalement industriel au ministère du Commerce et de l'Industrie. Ces écoles prennent le nom d'Écoles pratiques de commerce et d'industrie (E.P.C.I.).

-1er juin 1892 : décret plaçant sous l'autorité du ministère du Commerce et de l'Industrie 14 écoles primaires supérieures et professionnelles devenant E.P.C.I. -22 février 1893 : décret précisant les modalités de fonctionnement des E.P.C.I. -1893-1900 : le ministère du Commerce et de l'Industrie met en place toute une réglementation pour les E.P.C.I.

-2 avril 1900 : la Chambre des députés (24 novembre 1899) et le Sénat (2 avril 1900) votent le transfert des écoles nationales professionnelles au ministère du Commerce et de l'Industrie.

-27 décembre 1900 : loi plaçant les écoles professionnelles de la ville de Paris sous la tutelle du ministère du Commerce et de l'Industrie.

4ème période

-1901 : à l'initiative du Conseil supérieur du travail, enquête sur les conditions de l'apprentissage réalisée par l'office du travail.

-13 juillet 1905 : dépôt d'un projet de loi sur l'enseignement professionnel.

-28 juillet 1919 : loi Astier.

\section{NOTES}

1. Pierre Caspard (1989) analyse les raisons de cette situation qui reflète « le statut inférieur du technique dans l'enseignement comme dans la société ». Il passe en revue nos connaissances et nos lacunes.

2. Ponteil (1966) consacre quatre pages à cette question pour le XIX siècle et huit pages pour le $\mathrm{xx}^{\mathrm{e}}$ siècle sur un total de 454 pages; Prost (1968) lui accorde un chapitre de vingt-trois pages ; Mayeur (1981) évoque l'éducation technique (douze pages) et la naissance des grandes écoles techniques supérieures sous la Révolution ; Albertini (1992) « oublie » l'enseignement technique.

3. Folhen \& Bédarida (1959) ignorent l'histoire de l'enseignement technique. Le même constat s'applique aux ouvrages d'Alain Dewerpe (1989) et de Gérard Noiriel (1986).

4. Service d'histoire de l'éducation, unité de recherche associée au CNRS-URA 1397. Adresse postale : I.N.R.P.-C.N.R.S.-E.N.S., 45 rue d'Ulm, 75230 Paris Cedex 05. La recherche sur l'enseignement technique et professionnelle est animée depuis 1988 par Gérard Bodé.

5. À titre d'exemple, on citera les journées d'études organisées à l'Université de SaintEtienne en février 2003, dont les actes sont encours de publication.

6. Le programme et le calendrier des séances sont consultables sur Internet : www.inrp.fr/she/. Les séances se déroulent à l'INRP, 29 rue d'Ulm. 
7. Gérard Bodé, «Constitution d'un atlas-répertoire des établissements d'enseignement technique ", Histoire de l'éducation, n66 pp. 201-207, Gérard Bodé, Rapport de recherche. Je remercie Gérard Bodé de m'avoir communiqué ce texte.

8. Deux numéros publient des condensés des mémoires de maîtrise et DEA : Formations, emplois, XIX ${ }^{e}-X X^{e}$ siècles, 3-4/1997 et Métiers et statuts, 1-2/ 1999. Il serait souhaitable que les autres universités suivent cette exemple et qu'une base de données nationale relevant tous les mémoires de maîtrise consacrés à l'histoire de l'enseignement technique et professionnel.

9. Voir en particulier les pages 346-361, « les EPS des grandes villes entre l'enseignement secondaire et un enseignement pratique court ».

10. Cf. en particulier les journées d'études Istituzioni Formative e Agenti di Sviluppo nell' Italia septentionale (Secolo XIX-XX), organisées par le département d'Histoire de l'Université de Padoue les 25 et 26 janvier 2001. Une cinquantaine de communications y ont été présentées. Les actes seront publiés en 2006.

\section{RÉSUMÉS}

Pendant de nombreuses années, l'histoire de l'enseignement technique et de la formation professionnelle n'a pas été considérée. Ce n'est plus le cas, et le bilan des deux dernières décennies révèle que cette recherche est désormais un chantier en pleine activité, investi par des chercheurs venus d'horizons très différents. Cet article se propose de faire un état des lieux des travaux publiés. Il en ressort une tendance prometteuse à enrichir l'explication économique par la prise en compte de la dimension pédagogique des transformations du travail et de la production. Des zones d'ombre subsistent encore, attestant du caractère provisoire d'une histoire en construction.

Technological and professional teaching in France - 1800-1919.

For many years, attention has been paid neither to technological teaching nor professional training. This is not the case any more and during the last two decades this field is a priority for many researchers with very different backgrounds. This text will consider publications on the subject showing a promising tendency to strengthen economic explanation with the pedagogic dimension of transformation in work and production. Still some fields remain to be studied since construction of history is provisional.

La enseñanza técnica y profesional en Francia, 1800-1919. Un ensayo de balance historiográfico.

Por largo tiempo la historia de la enseñanza técnica y de la formación profesional no ha recibido atención. Esto ha cambiado, y un balance de las dos últimas décadas muestra que la investigación en este campo vive un periodo de efervescencia, animado por investigadores provenientes de horizontes muy diversos. Este artículo se propone hacer un estado de la cuestión de los trabajos publicados. Destaca una tendencia prometedora a enriquecer la explicación económica tomando en consideración la dimensión pedagógica de las transformaciones del trabajo y de la producción. Persisten aún ciertas zonas de sombra, atestiguando que nos encontramos ante una historia en proceso de construcción. 
INDEX

Mots-clés : histoire, enseignement technique, formation professionnelle

Keywords : history, professional formation, technical teaching

AUTEUR

PHILIPPE MARCHAND

Université Charles-de-Gaulle - Lille 3, UMR 8529 CERSATES. 